\section{Ariadne Genomics Announces the Release of Seqware Data Center Self-Updating Sequence Data Management and Personal Blast System}

ROCKVILLE, MD (PRWEB) (February 7, 2005) - Seqware Data Center includes powerful database and fast, fully functional Blast server. It is a scalable solution that helps individual scientists, workgroups and core facilities manage their proprietary DNA sequences along with public sequence collections on a local PC or a network. The amount of sequence data efficiently stored by Seqware is limited by disk space only.

Seqware automatically updates GenBank from NCBI data repository, and makes it easy to create, manage, update and annotate sequence sets, edit and annotate sequence records in the database, run flexible context searches, store, automatically update and reuse Blast results.

'Seqware Data Center can serve as a powerful research tool for an individual scientist, and as principal sequence data repository in Bioinformatics departments and core facilities,' said Dr. Anton Yuryev, Director of Application Science at Ariadne Genomics. 'With its simple traditional interface, powerful database and affordable pricing, Seqware is a 'must have' solution for all scientists who work with sequences on a regular basis.

Seqware Data Center installs seamlessly from a set of CD/DVD ROMs and requires no specific maintenance or da- tabase administration. No additional database licenses are required.

For more information about Seqware Data Center, visit Ariadne Genomics website www.ariadnegenomics.com/ products/seqware.html.

\section{About Ariadne Genomics}

Ariadne Genomics is a Marylandbased company developing systems biology tools for life science research: Pathway Studio Suite for reconstruction, analysis and visualization of biological pathways; MedScan TextMiner, an automated scientific text processing tool; ResNet, a comprehensive molecular networks database, created by automated extraction of biological facts from the entire PubMed, and Seqware, a personal Blast and sequence data management platform.

Natalia Alexandrova

ARIADNE GENOMICS

natal@ariadnegenomics.com

8476441557

www.ariadnegenomics.com

\section{Signature Genomic Laboratories, LLC further expand its clinical cytogenetic services with the addition of FISH and Karyotyping}

Following the success of the Signature$\mathrm{Chip}^{\mathrm{TM}}$ and SignatureSelect microarray services, a new approach to cytogenetic analysis by screening for multiple clinical loci, Signature Genomic Laboratories is pleased to announce further expansion in its cytogenetic services.

Signature Genomic Laboratories launched their unique microarray analysis services in March of 2004 with the innovative SignatureChip microarray which analyses 126 clinical loci in one experiment with a report time of 5 days. Four months later, Signature launched a customized microarray service, allowing the clinician to customize a microarray using any of the 126 clinical loci available from Signature. FISH and Karyotyping have now been added to Signature's portfolio, enabling Signature to offer the complete range of clinical cytogenetic services that clinicians demand.

Lisa G. Shaffer, Ph.D., Technical Laboratory Director commented: "I'm excited to be able to offer the clinician the flexibility to order exactly what they need from a single laboratory".

Bassem Bejjani, M.D., Medical Director added: "we always listen to our customers. Many informed us that they wanted to send their patients' samples to a single laboratory for traditional chromosome analysis and then have the option of adding array analysis, should the karyotype be normal. This is why we established this service. This service makes perfect sense from a medical and business perspective. We look forward to additional input from our customers and colleagues".

Located in Spokane, WA, Signature Genomic Laboratories is the leader in cytogenetic analysis services using microarray based technologies. This revo-

\section{Normal Chromosome 15}

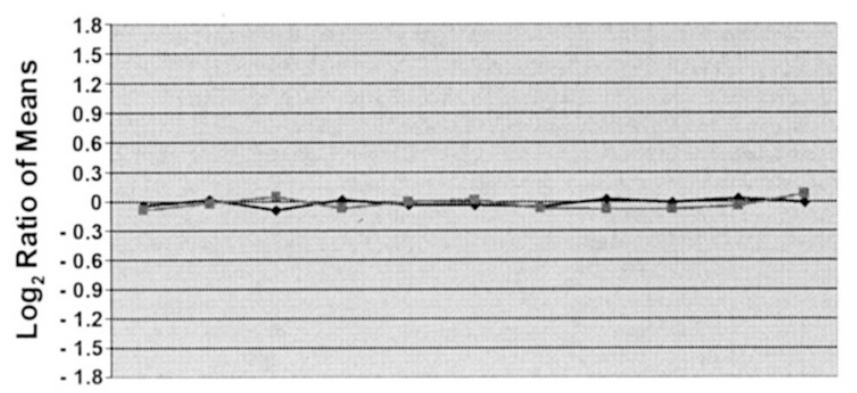

$\operatorname{dup}(15)(q 12 q 12)$

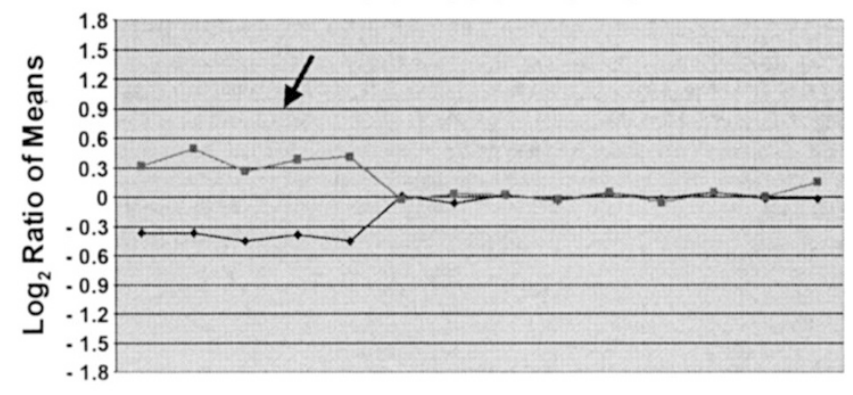

Typical result from SignatureChip ${ }^{\mathrm{TM}}$ microarray clinical cytogenetic service. In this case, SignatureChip analysis identified a duplication of the SNRPN/UBE3A locus at $15 \mathrm{q} 12$. 
lutionary approach offers clinicians and patients the opportunity to diagnose cytogenetic disorders in a fast, reliable, and comprehensive format.

Additional information on Signature Genomic Laboratories cytogenetic services can be found at www. signaturegenomics.com
Spencer Howell

Marketing Director

HOWELL@signaturegenomics.com

The "New Products" page is designed to offer you news and information from businesses serving the genetics community. We welcome your submissions. All submissions are subject to review by the Editor. For more information, contact Al Lucchesi, National Accounts Manager, Lippincott Williams \& Wilkins, 530 Walnut Street, Philadelphia, PA 19106; phone 215-521-8409; fax 215-521-8411; emailalucches@lww.com. 